\title{
Metallentfernung nach Schenkelhalsfraktur
}

\author{
Felix Bonnaire
}

Am 8.7.2004 um 18.00 Uhr stürzte ein 65-jähriger Autohändler beim InlineSkaten auf die linke Hüfte und wurde in der Notaufnahme unseres Hauses vorgestellt. Die klinische Diagnostik ergab den Verdacht auf eine proximale Oberschenkelfraktur links, wesentliche Begleiterkrankungen lagen nicht vor.

In der Röntgendiagnostik zeigte sich eine dislozierte intrakapsuläre Schenkelhalsfraktur, die als Garden III eingestuft wurde (Abb.1).

Die Indikation zur gelenkerhaltenden Osteosynthese wurde sofort gestellt und Herr G. wurde zeitnah um 1.00 Uhr vom diensthabenden Oberarzt mit einer DHS und Antirotationsschraube versorgt.

Die intraoperativen fluorografischen Bilder zeigten zunächst eine anatomische Reposition (Abb.2), die Aufnahmen nach Osteosynthese dokumentieren eine neu aufgetretene Diastase an der Bruchfläche, einen Versatz des Kopffragments nach kaudal am Adam'schen Bogen und eine neu aufgetretene Antekurvation der Schenkelhalsachse, verbliebener Retroversion des Kopfes und mit einer daraus resultierenden Lage der Schenkelhalsschraube im vorderen oberen Quadranten (Abb. 3).

Die Situation wurde vom Operateur als belastungsstabil eingeschätzt und am 2. postoperativen Tag wurde mit der Mobilisation unter Vollbelastung begonnen. Die postoperativen Röntgenaufnahmen vor der Entlassung zeigten den intraoperativ bereits konstatierten Kaudalversatz des Kopffragments und dessen leichte Abkippung nach dorsal. Die Diastase war durch die Belastung verschwunden, der Schenkelhals eher ver-

OP-JOURNAL 2008; 24: 226-228

(c) Georg Thieme Verlag KG Stuttgart • New York DOI 10.1055/s-2008-1039073

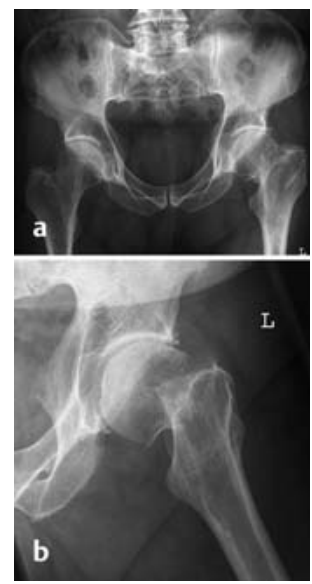

Abb. 1 a und $b$ a Beckenübersicht, $\mathbf{b}$ axiale Aufnahme.

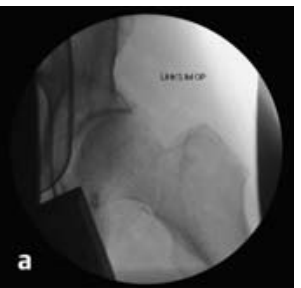

Abb. $2 \mathrm{a}$ und $\mathbf{b}$ Intraoperativ gut reponierte Schenkelhalsfraktur im a.-p. (a) und axialen Strahlengang (b).

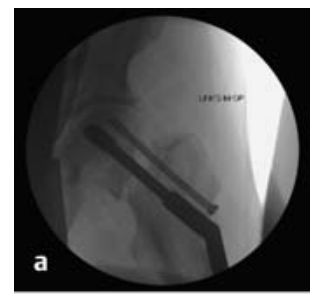

Abb. $3 \mathbf{a}$ und $\mathbf{b}$ Nach Einbringen der DHS und der Antirotationsschraube zeigt sich a.-p. (a) eine geringe Diastase der Fraktur und axial (b) eine wieder aufgetretene Retrotorsion des Kopffragments.
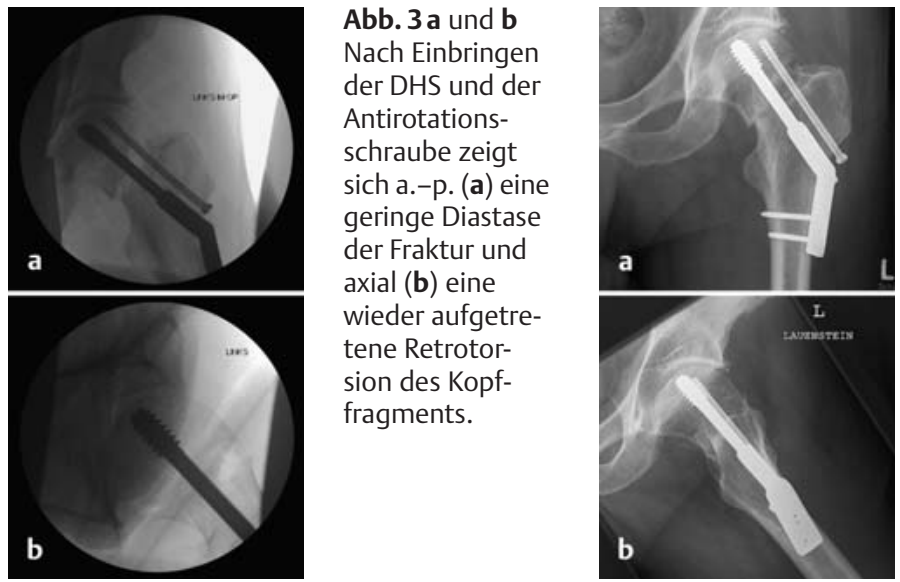

Abb. $4 \mathrm{a}$ und $\mathrm{b}$ Die postoperativen Aufnahmen zeigen eine Impaktierung und einen Kaudalversatz des Kopffragments in der a.-p. Ebene (a) nach Belastung und die verbliebene geringe Retrotorsion des Kopfes.

kürzt. Der Patient hat die üblichen geringen Beschwerden bei der Belastung und wurde in eine Rehaklinik verlegt (Abb.4). Es war von einem beobachtungswürdigen Verlauf auszugehen.

Am 27.1.2005 stellte sich Herr G. mit geringen Beschwerden an der linken Hüfte vor. Er habe noch einen watschelnden Gang und ein Reiben über der Außenseite am Trochanter major. Die Röntgenaufnahmen demonstrieren einen verheilten Schenkelhalsbruch mit verkürztem Schenkelhals und nach lateral heraustretender Schenkelhalsschraube
(Abb.5). Die Beschwerden konnten auf die beschriebenen Röntgenveränderungen zurückgeführt werden und ich empfahl eine Metallentfernung. Der Patient wünschte jetzt die Behandlung durch den Chefarzt, also mich. Jetzt beginnt meine Geschichte: Die Metallentfernung wurde am 2. März 2006, also 8 Monate nach der Osteosynthese, durch mich durchgeführt und verlief problemlos.

Am 2. postoperativen Tag klagte Herr G. nach der Vollbelastung über einen Schmerz in der Leiste und ein Unsicherheitsgefühl und Schwäche im linken 


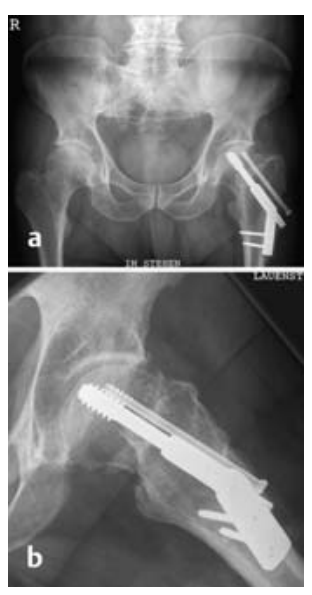

Abb. 5 a und $\mathbf{b}$ Die vor der geplanten Metallentfernung angefertigten Röntgenbilder wurden wie folgt interpretiert: in der a.-p. Ebene in Kaudalversatz und Verkürzung verheilte Fraktur (a) und in der axialen Ebene in leichter Retrotorsion verheilte Schenkelhalsfraktur (b).

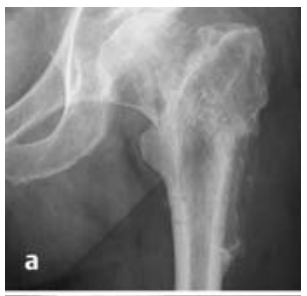

Abb. $6 \mathrm{a}$ und $\mathbf{b}$ Die Röntgenbilder nach Metallentfernung zeigen a.-p. und axial eine dislozierte Pseudarthrose nach Belastungswiederaufnahme durch den Patienten. Der Schenkelhals ist stark verkürzt.

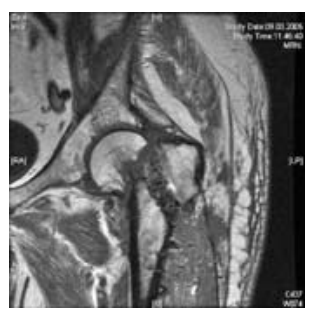

Abb. 7 a und $\mathbf{b}$ MRT-Darstellung der erhaltenen Femurkopfdurchblutung.

Bein. Die Röntgenaufnahme zeigte eine Schenkelhalspseudarthrose (Abb.6). Zur Therapieplanung wurde eine MRT-Aufnahme veranlasst, um die Perfusionsverhältnisse zu analysieren (Abb. 7).

Die Situation wurde mit dem Patienten erörtert, und aufgrund der zu erwartenden verbleibenden Verkürzung des Schenkelhalses einigten wir uns auf eine zementfreie Totalendoprothese zur Lösung des Problems. Dabei sollte die Beinlängendifferenz ausgeglichen werden.

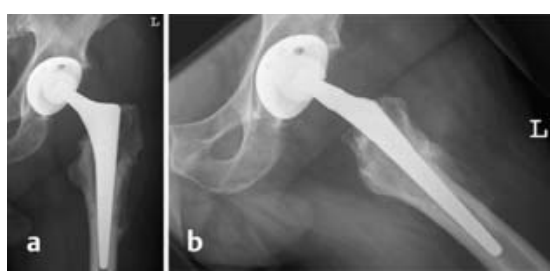

Abb. 8a und b Postoperative Aufnahme nach Implantation einer zementfreien TEP. Die Prothese ist nicht weit genug im Schaft verankert (a): es resultiert ein vergrößertes Offset. Auch axial (b) ist dies zu erkennen.

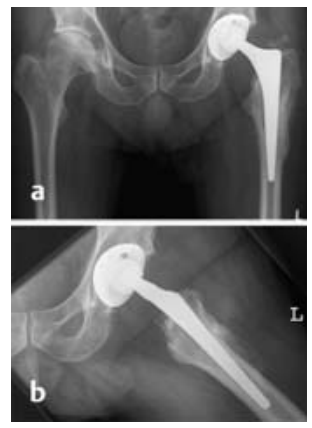

Abb. 9 a und $\mathbf{b}$ Kontrolle nach 3 Monaten: unveränderte Stellung unter Vollbelastung.

Intraoperativ war es schwierig, die angestrebte Verlängerung zu erlangen und den Prothesenschaft wie zuvor ausgemessen in der benötigten Stärke in den Oberschenkelschaft einzuschlagen. Ich befürchtete eine Schaftsprengung oder Trochterfraktur bei tieferem Einschlagen der an sich sehr fest sitzenden kleinstmöglichen Schaftkomponente (Abb.8). Die Beinlänge wurde dabei ausgeglichen und ich erhoffte noch einen gewissen Setzvorgang.

Herr G. ging wieder zur Reha und wurde von mir nachbehandelt. Nach 3 Monaten war die Röntgenkontrolle unverändert (Abb.9) und Herr G. durfte wieder Wanderungen machen. Er hatte nur „Muskelkater“, allerdings nach längerem Laufen einen watschelnden Gang, wie er sagte.

Nach dem Sommer teilte mir der weiterbehandelnde Orthopäde einen CRPWert von $32,7 \mathrm{mg} / \mathrm{ml}$ mit, Beschwerden lagen nur mäßige vor. Bei Kontrollen bestätigte sich der CRP-Wert und eine Punktion des Hüftgelenks und Szintigrafie brachte weder einen Keimnachweis noch eine sichere Lockerung.

Eine Kontrolluntersuchung am 24.4. 2006, also etwa 1 Jahr nach dem Unfall, zeigte einen Setzvorgang der femoralen Prothesenkomponente um $2,5 \mathrm{~cm}$ (Abb.10). Ein Beinlängenausgleich

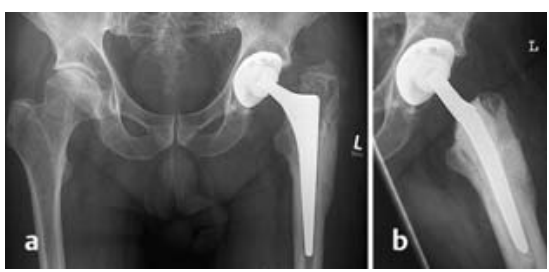

Abb. 10 a und b Ein Jahr nach TEP-Implantation deutlich erkennbarer Setzvorgang der Prothese mit klinischer Lockerung.

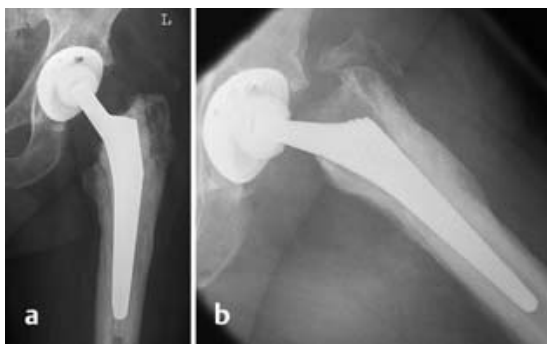

Abb. 11 a und b Bilder nach Schaftwechsel und Inlay-Wechsel der Pfanne. Umstieg auf eine zementierte Geradschaftprothese.

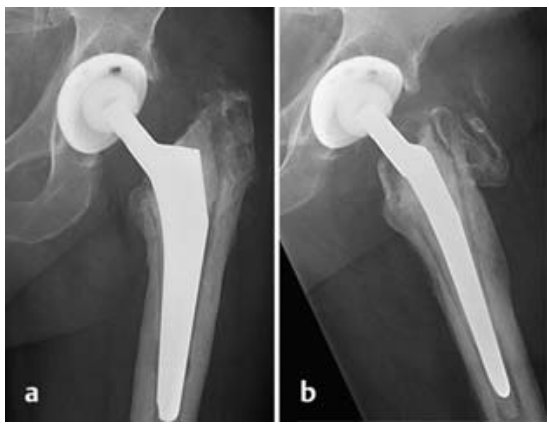

Abb. 12 a und $\mathbf{b}$ Aufnahmen nach $1 \mathrm{Jahr}$ zeigen deutlichen Lysesaum um den Schaft bei eindeutigem Infektnachweis.

durch Schuherhöhung konnte das Laufen verbessern, lange Zeit lag nahezu Beschwerdefreiheit vor. CRP-Kontrollen lagen zum Teil bei 5, dann bei $70 \mathrm{mg} / \mathrm{ml}$.

Eine Punktion des Hüftgelenks ergab am 3.4.2006 eine Infektion mit koagulasenegativen Staphylokokken und Staphylococcus capitis. Ich stellte die Indikation zur Wechseloperation. Der Patient wünschte einen einzeitigen Wechsel, den ich auch befürwortete. Ich habe das Inlay gewechselt und eine Geradschaftprothese mit Refobacin-Vancomycin Palacos einzeitig eingebaut (Abb.11). Die Wundheilung war unkompliziert, die Mobilisation gelang gut. Die CRPWerte entwickelten sich zu Normwerten. Nach einem Jahr stellten sich wie- 


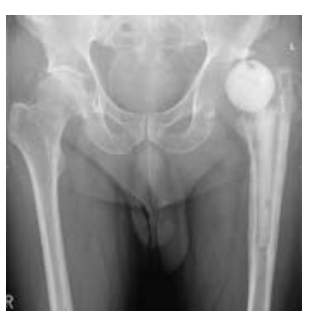

Abb. 13

Beckenübersicht nach der Explantation der Pfanne und des Schaftes und Einsatz eines antibiotikahaltigen Spacers.

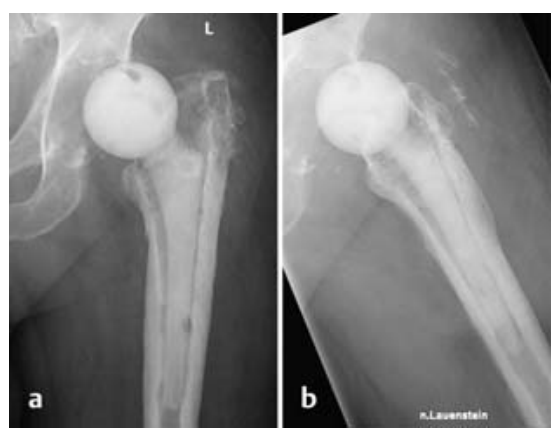

Abb.14a und b Aufnahmen der Hüfte 3 Monate nach Einbringen des Spacers. Subluxation, aber gute Mobilisation.

der Schmerzen und erhöhte Entzündungsparameter ein.

Eine erneute Entzündungsdiagnostik ergab keinen Nachweis von Keimen, die Beschwerden wechselten und zuletzt, im Januar 2008, waren sie kontinuierlich, wenn auch nicht sehr stark.

Die Röntgenaufnahmen zeigten jetzt eine eindeutige Lockerung der Schaftkomponente (Abb.12). Eine Punktion hatte wiederum Staphylococcus capitis

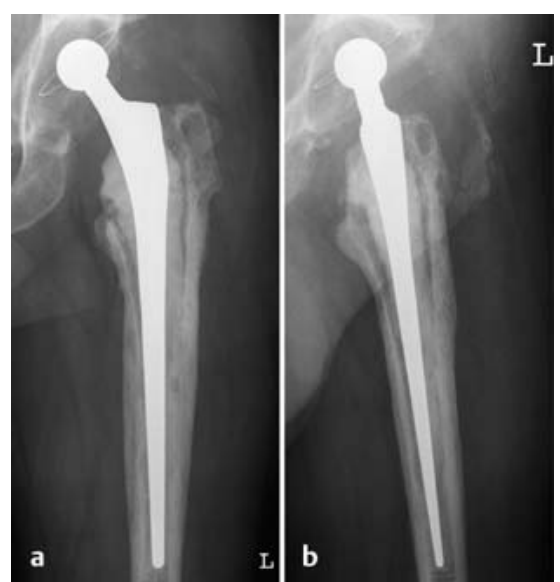

Abb. 15a und b Letzte Röntgenbilder nach Implantation einer zementierten Langschaftprothese mit zementierter Pfanne.

und zusätzlich vergrünende Streptokokken. Jetzt beschloss ich, alle Implantate einschließlich Zement zu entfernen und einen Spacer mit resistenzgerechtem Antibiotikazusatz zu implantieren (Abb.13). Herr G. konnte zufriedenstellend mobilisiert werden (Abb.14).

Vier Monate nach Spacerimplantation und bei CRP-Werten im Normbereich habe ich eine Langschaftprothese mit Refobacin Palacos und resistenzgerechtem Antibiotikumzusatz implantiert (5. Operation, Abb.15). Keime waren intraoperativ nicht mehr nachzuweisen. Die Entzündungswerte normalisierten sich nach der Operation, der Patient konnte wieder mobilisiert werden.
Fazit

Die Komplikationsreihe setzt sich aus verschiedenen Schritten zusammen: Aus meiner retrospektiven Betrachtung war die Osteosynthese am Schenkelhals dezent misslungen und für Probleme prädestiniert, und vor der Metallentfernung wäre eine CT-Untersuchung angebracht gewesen. Die erste zementfreie Prothese saß nicht korrekt im Schaft, ich hätte sie noch weiter aufraspeln müssen. Die Hoffnung auf einen Setzvorgang war trügerisch. Er trat ein, allerdings in Verbindung mit einer septischen Lockerung. Der Patientenwunsch nach kontinuierlicher Mobilität verführte mich zu einem einzeitigen Wechsel, der nicht gutging und zu einer erneuten septischen Lockerung führte. Jetzt war ein konfektionierter Spacer die Methode der Wahl und ein zweizeitiger Wechsel angemessen, danach die ReReTEP. Ob der Infekt definitiv beseitigt ist, kann ich noch nicht beantworten. Mittlerweile habe ich den Patienten 4-mal operiert, zusammen mit der 1 . Operation hat er 5 Operationen in 4 Jahren und 4 Rehaaufenthalte hinter sich. Mein persönliches Verhältnis zu ihm hat sich glücklicherweise eher intensiviert als verschlechtert. On verra.

\section{Prof. Dr. med. Felix Bonnaire}

Chefarzt

Städtisches Krankenhaus

Dresden-Friedrichstadt

Friedrichstraße 41

01326 Dresden

E-Mail: bonnaire-fe@khdf.de 